\title{
Stabilization of Discrete-Time Piecewise Affine Systems in Implicit Representation
}

\author{
L. Cabral $^{1}$, J. M. Gomes da Silva Jr. ${ }^{1}$, G. Valmorbida ${ }^{2}$
}

\begin{abstract}
This paper addresses the problem of stabilization of discrete-time piecewise affine (PWA) systems. The design of a piecewise affine state feedback control law is studied using an implicit representation based on ramp functions. LMI-based stability conditions, obtained from a piecewise quadratic Lyapunov function and the implicit representation, are stated to assess the global exponential stability of the origin of the closed-loop PWA system. Through appropriate congruence transformations and some structural assumptions, a method to design the control law parameters using semidefinite programming is then proposed.
\end{abstract}

\section{INTRODUCTION}

Piecewise Affine (PWA) systems have been used to model nonlinear circuits [12] and other physical systems [14]. They can also represent some classes of switched and hybrid systems [3]. Moreover, some nonlinear functions that arise in dynamic control systems can also be modeled or approximated by PWA functions.

Many different forms to represent PWA systems have been proposed in the literature [6]. The most common and intuitive representation of a discrete-time PWA system is given by the explicit representation [13]

$$
x^{+}=A_{i} x+a_{i}, \forall x \in \Gamma_{i}
$$

where $\Gamma_{i}$ is the $i$ th set in the partition of the state space, described in general by a finite number of explicit inequalities, $x$ and $x^{+} \in \mathbb{R}^{n}$ are, respectively, the current and the successor state, matrix $A_{i} \in \mathbb{R}^{n \times n}$ and vector $a_{i} \in \mathbb{R}^{n}$ defines the dynamical behavior of the system in region $\Gamma_{i}$. In particular, if the vector field is continuous over the boundary of the partition, we refer to this class as discrete-time Continuous PWA (CPWA) system. Moreover, if $\cup_{i=1}^{N} \Gamma_{i}=\mathbb{R}^{n}$, where $N$ is the number of sets in the partition, the representation in (1) is globally valid.

Considering the above explicit representation, many conditions to assess the stability of discrete-time PWA systems have been proposed in the literature. We can cite, for instance, [3] and [2]. In this case, to evaluate the decrease of the Lyapunov function along the trajectories of the system,

\footnotetext{
${ }^{1}$ L. Cabral and J. M. Gomes da Silva Jr. are with PPGEE Programa de Pós-Graduação em Engenharia Elétrica, Universidade Federal do Rio Grande do Sul, Porto Alegre, RS, Brazil. leonardo.cabraldufrgs.br, jmgomes@ufrgs.br.

${ }^{2}$ G. Valmorbida is with Laboratoire des Signaux et Systèmes, CentraleSupèlec, CNRS, Université Paris-Saclay, 91192, Gif-sur-Yvette, France, and also with INRIA Saclay, 91120, Palaiseau, France. giorgio.valmorbida@l2s.centralesupelec.fr

This study was financed in part by the Coordenação de Aperfeiçoamento de Pessoal de Nível Superior - Brasil (CAPES) - Finance Code 001, by the Brazilian National Council for Research (CNPq) - Grant 307449/2019-0 and by the ANR project HANDY under grant ANR-18-CE40-0010.
}

the possible transitions between regions must be taken into account. In general, all the possible transitions are tested, introducing some conservatism in the conditions. An analysis to determine the possible regions that can be reached from a given set in the partition helps reduce the conservatism in the analysis [1], even though the number of transitions can present combinatorial growth as the number of sets in the partition increases.

To overcome the need for the preliminary reachability analysis, an implicit representation, based on the use of ramp functions, has been recently proposed in [4]. From this representation, it is possible to express piecewise quadratic Lyapunov candidate functions in a compact way and to cast Lyapunov conditions as generalized quadratic inequalities involving ramp functions and their arguments. Furthermore, based on an exact description of the ramp function in terms of linear inequalities and a quadratic identity, it is possible to check these generalized quadratic inequalities using LMIs.

The problem of stabilization has been studied using the explicit representation. For continuous-time PWA systems, [5] casts the design of a piecewise linear state feedback control law as a convex optimization problem, but this is achieved by the restrictive assumption of a common quadratic Lyapunov function to all sets in the partition. In [12] the synthesis of a piecewise affine feedback control law is formulated as an optimization problem subject to a set of quasi-LMIs. However, the method only applies to slab continuous-time PWA systems. For discrete-time systems, [9] considers a piecewise quadratic Lyapunov function to obtain conditions for the synthesis of a piecewise linear state feedback control law in terms of a convex optimization problem. However, it should be noticed that the presented stabilization condition applies only when the system is piecewise linear. Due to the affine term, the extension to the PWA case is not as straightforward. Furthermore, as the explicit representation (1) is used, all the possible transitions from one region to another must be considered. This comes from the fact that the closed-loop gains are unknown and thus a reachability analysis cannot be performed to reduce the needed tests and the conservatism. Moreover, diferently from the analysis conditon (see also [7]), the fact that a transition from partition $j$ to $i$ can happen only if $x \in \Gamma_{j}$ is not taken into account, which is another source of conservatism.

Thanks to the advantages of the representation presented in [4], this paper investigates the global stabilization problem using this implicit representation. With this aim, starting from the Lyapunov inequalities formulated for the stability analysis, we obtain quasi-LMI conditions allowing the 
synthesis of stabilizing gains through the solution of semidefinite programming problems.

Notation: For a vector $v \in \mathbb{R}^{n}, v_{i}$ denotes its $i$ th element and $v \succeq 0(v \preceq 0)$ denotes elementwise nonnegativity (nonpositivity). For a matrix $M \in \mathbb{R}^{n \times m}, M_{(i, j)}$ denotes its $(i, j)$ element while $M_{i j}$ denotes its $(i, j)$ block. Moreover, $M>0(M \geq 0)$ denotes a positive (semi) definite matrix, $M \succeq 0$ denotes an elementwise nonnegative matrix, $\|M\|$ denotes the largest singular value of $M$ and $\operatorname{He}\{M\} \triangleq M+$ $M^{T}$. The set of diagonal matrices in $\mathbb{R}^{n \times n}$ is represented by $\mathbb{D}^{n}$.

\section{Problem Statement}

Consider a discrete-time PWA system, described with the implicit representation proposed in [4] as

$$
\begin{aligned}
& x^{+}=F_{1 o} x+F_{2 o} \phi(y(x))+B u \\
& y(x)=F_{3} x+F_{4} \phi(y(x))+f_{5},
\end{aligned}
$$

where $x \in \mathbb{R}^{n}$ is the state, $u \in \mathbb{R}^{n_{u}}$ is the control input and $y \in \mathbb{R}^{n_{y}}$ is the argument to the vector-valued ramp function $\phi: \mathbb{R}^{n_{y}} \rightarrow \mathbb{R}^{n_{y}}$, which is defined elementwise in terms of the ramp function $r: \mathbb{R} \rightarrow \mathbb{R}$ as

$$
\phi_{i}(y)=r\left(y_{i}\right)=\left\{\begin{array}{l}
0 \text { if } y_{i}<0 \\
y_{i} \text { if } y_{i} \geq 0
\end{array}\right.
$$

for each $i=1, \ldots, n_{y}$. The system dynamics (2) is defined by the constant matrices $F_{1 o} \in \mathbb{R}^{n \times n}, F_{2 o} \in \mathbb{R}^{n \times n_{y}}, B \in$ $\mathbb{R}^{n \times n_{u}}, F_{3} \in \mathbb{R}^{n_{y} \times n}, F_{4} \in \mathbb{R}^{n_{y} \times n_{y}}$ and vector $f_{5} \in \mathbb{R}^{n_{y}}$.

Equation (2b) defines the activation of affine terms through function $\phi$ depending on the state value. Hence, there is a direct relation of this equation and the partition regions $\Gamma_{i}$ of an explicit representation (see [4] for details). In particular, for each region of the explicit representation, some entries of $\phi$ will be zero and others will be equal to $y_{i}$. For example, consider the nonlinear circuit presented in [12] and shown in Figure 1. The nonlinear resistor characteristic is modeled by a continuous piecewise affine function as depicted in Figure 2, from where we notice the existence of three equilibrium points, one in each set of the partition.

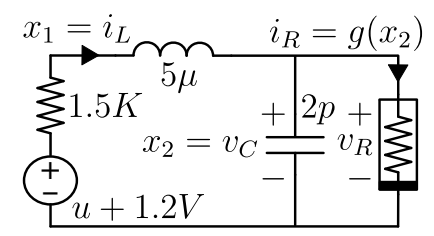

Fig. 1. Circuit with nonlinear resistor [12].

For a sampling period $T$, a discrete-time explicit PWA representation for the behavior of the system is given by (1)

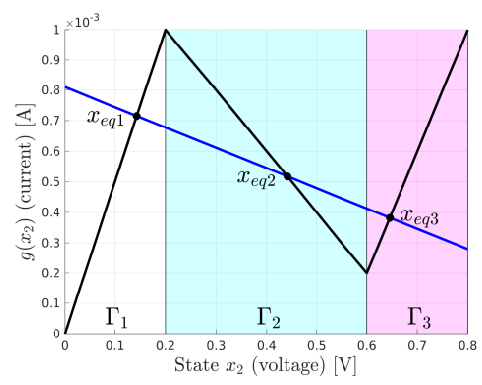

Fig. 2. Piecewise affine characteristic of the nonlinear resistor (black) and load given by the $1.5 \mathrm{~K}$ resistor (blue) in Figure 1 .

with 3 regions, as follows:

$$
\begin{aligned}
& A_{1}=\left[\begin{array}{cc}
1-30 T & -20 T \\
T / 20 & 1-T / 4
\end{array}\right], a_{1}=\left[\begin{array}{c}
24 T \\
0
\end{array}\right] \\
& A_{2}=\left[\begin{array}{cc}
1-30 T & -20 T \\
T / 20 & 1+T / 10
\end{array}\right], a_{2}=\left[\begin{array}{c}
24 T \\
-0.07 T
\end{array}\right] \\
& A_{3}=\left[\begin{array}{cc}
1-30 T & -20 T \\
T / 20 & 1-T / 5
\end{array}\right], a_{3}=\left[\begin{array}{c}
24 T \\
0.11 T
\end{array}\right], B=\left[\begin{array}{c}
20 T \\
0
\end{array}\right], \\
& \Gamma_{1}=\left\{x \in \mathbb{R}^{2} \mid\left[\begin{array}{cc}
0 & -1
\end{array}\right] x \geq-0.2\right\} \\
& \Gamma_{2}=\left\{x \in \mathbb{R}^{2} \mid\left[\begin{array}{cc}
0 & 1 \\
0 & -1
\end{array}\right] x \succeq\left[\begin{array}{c}
0.2 \\
-0.6
\end{array}\right]\right\} \text { and } \\
& \Gamma_{3}=\left\{x \in \mathbb{R}^{2} \mid\left[\begin{array}{ll}
0 & 1
\end{array}\right] x \geq 0.6\right\} .
\end{aligned}
$$

An implicit representation of the same circuit is given by (2a) and (2b) with

$$
\begin{aligned}
& F_{1 o}=\left[\begin{array}{cc}
1-30 T & -20 T \\
0.05 T & 1-0.25 T
\end{array}\right], F_{2 o}=\left[\begin{array}{ccc}
T & 0 & 0 \\
0 & 50 T & -50 T
\end{array}\right], \\
& B=\left[\begin{array}{c}
20 T \\
0
\end{array}\right], F_{3}=\left[\begin{array}{cc}
0 & 0 \\
0 & 0.007 \\
0 & 0.006
\end{array}\right], F_{4}=0, f_{5}=\left[\begin{array}{c}
24 \\
-0.0014 \\
-0.0036
\end{array}\right] .
\end{aligned}
$$

Note that the partition is defined by the sets

$$
\begin{aligned}
& \Gamma_{1}=\left\{x \in \mathbb{R}^{n} \mid y_{1} \geq 0, y_{2}<0, y_{3}<0\right\}, \\
& \Gamma_{2}=\left\{x \in \mathbb{R}^{n} \mid y_{1} \geq 0, y_{2} \geq 0, y_{3}<0\right\}, \\
& \Gamma_{3}=\left\{x \in \mathbb{R}^{n} \mid y_{1} \geq 0, y_{2} \geq 0, y_{3} \geq 0\right\} .
\end{aligned}
$$

For $x \in \Gamma_{i}$, we have $\phi(y(x))=\Phi_{i} y(x)$, where matrices $\Phi_{i} \in \mathbb{D}^{n_{y}}$ have the diagonal elements equal to one corresponding to the nonnegative elements in vector $y$, and zero otherwise. For the previous example, the matrices are

$$
\Phi_{1}=\left[\begin{array}{lll}
1 & 0 & 0 \\
0 & 0 & 0 \\
0 & 0 & 0
\end{array}\right], \Phi_{2}=\left[\begin{array}{lll}
1 & 0 & 0 \\
0 & 1 & 0 \\
0 & 0 & 0
\end{array}\right] \text { and } \Phi_{3}=\left[\begin{array}{lll}
1 & 0 & 0 \\
0 & 1 & 0 \\
0 & 0 & 1
\end{array}\right] .
$$

Hence, the relation between the explicit representation (1) and the implicit representation (2) is given by

$$
\begin{aligned}
& A_{i}=F_{1 o}+F_{2 o} \Phi_{i}\left(I-F_{4} \Phi_{i}\right)^{-1} F_{3} \\
& a_{i}=F_{2 o} \Phi_{i}\left(I-F_{4} \Phi_{i}\right)^{-1} f_{5} .
\end{aligned}
$$

In this work, we are interested in computing a stabilizing control law $u$ such that the global exponential stability of the origin of the closed-loop system is ensured. With this 
aim, the following nonlinear state feedback control law is considered:

$$
u(x)=K_{1} x+K_{2} \phi(y(x))
$$

with $K_{1} \in \mathbb{R}^{n_{u} \times n}$ and $K_{2} \in \mathbb{R}^{n_{u} \times n_{y}}$. The closed-loop system composed by (2) and (6) reads:

$$
\begin{aligned}
& x^{+}=F_{1} x+F_{2} \phi(y(x)) \\
& y(x)=F_{3} x+F_{4} \phi(y(x))+f_{5}
\end{aligned}
$$

where $F_{1}=\left(F_{1 o}+B K_{1}\right)$ and $F_{2}=\left(F_{2 o}+B K_{2}\right)$. Note that the gain $K_{2}$ modifies the control action according to the active set $\Gamma_{i}$.

Regarding representation (7) of the closed-loop system, the following assumption is considered.

Assumption 1. The algebraic loop in (7b) is well-posed, i.e. there is an unique solution to the implicit equation (7b).

Assumption 1 ensures the existence of inverse for $(I-$ $\left.F_{4} \Phi_{i}\right)$ in (5) and can be verified by applying the following result, whose proof is found in [4].

Proposition 1. If there exists matrix $X \in \mathbb{D}^{n_{y}}$ such that

$$
-2 X+X F_{4}+F_{4}^{T} X<0
$$

then the implicit equation (2b) is well-posed.

\section{PROPERTIES OF RAMP FUNCTIONS}

Based on properties of the ramp function, this section states two lemmas for the function $\phi$, which will be instrumental to obtain conditions for the stability and stabilization of the closed-loop system.

Since $\phi$ is defined elementwise in terms of a ramp function, it inherits the following properties from the ramp function, valid for any vector $y \in \mathbb{R}^{n_{y}}[11]$ :

$$
\begin{aligned}
& \phi_{i}(y) \geq 0 \\
& \left(\phi_{i}(y)-y_{i}\right) \geq 0 \\
& \phi_{i}(y)\left(\phi_{i}(y)-y_{i}\right)=0 .
\end{aligned}
$$

It should be noticed that these relations apply only to ramp functions, and so its use to obtain stability conditions does not introduce any conservatism. This is a key difference with respect to sector-bounded relations [8], which applies to a broad class of functions.

Let $\xi^{T}(y) \triangleq\left[\begin{array}{lll}1 & \phi^{T}(y) & (\phi(y)-y)^{T}\end{array}\right]$. Based on properties given in $(8)$, the following lemmas can be stated.

Lemma 1. For any symmetric elementwise nonnegative matrix $M=M^{T} \succeq 0 \in \mathbb{R}^{\left(1+2 n_{y}\right) \times\left(1+2 n_{y}\right)}$ and $y \in \mathbb{R}^{n_{y}}$, the function $\phi$ defined in (3) is such that

$$
s_{1}(M, y) \triangleq \xi^{T}(y) M \xi(y) \geq 0 .
$$

Proof. From (8a) and (8b) all elements of the vector $\xi(y)$ are nonnegative. Since each element of $M$ is nonnegative, then (9) holds.
Lemma 2. For any $T \in \mathbb{D}^{n_{y}}, y \in \mathbb{R}^{n_{y}}$ and the function $\phi$ defined in (3), it follows that

$$
s_{2}(T, y) \triangleq \xi(y)^{T}\left[\begin{array}{ccc}
0 & 0 & 0 \\
0 & 0 & T \\
0 & T & 0
\end{array}\right] \xi(y)=0 .
$$

Proof. Since $T$ is a diagonal matrix, expression $s_{2}(T, y)$ can be written as $s_{2}(T, y)=2 \sum_{i=1}^{n_{y}} T_{(i, i)} \phi_{i}(y)\left(\phi_{i}(y)-y_{i}\right)$, which is equal to zero as a consequence of property $(8 \mathrm{c})$.

\section{Stability CONDitions}

The next two lemmas result from the relation between $y$ and $x$ in (7) and are important to state the stability conditions. Let $\chi^{T}(x) \triangleq\left[\begin{array}{llll}1 & x^{T} & \phi^{T}(y(x)) & (\phi(y(x))-y(x))^{T}\end{array}\right]$.

Lemma 3. For any vector $\zeta \in \mathbb{R}^{n_{\zeta}}$ and matrix $R \in \mathbb{R}^{n_{\zeta} \times n_{y}}$ the relation

$$
s_{3}(R, \zeta, x) \triangleq \zeta^{T} R Q \chi(x)=0,
$$

with $Q=\left[\begin{array}{llll}f_{5} & F_{3} & F_{4}-I & I\end{array}\right]$ is verified along the trajectories of the closed-loop system (7).

Proof. Note that from (7b) we have $Q \chi(x)=0 \forall x \in \mathbb{R}^{n}$.

It is possible to state an extended version of Lemma 3 using an augmented form of vector $\chi(x)$ defined as

$$
\tilde{\chi}^{T}(x)=\left[\begin{array}{lllll}
1 & x^{T} & \left(x^{+}\right)^{T} & \phi^{T}(\tilde{y}(x)) & (\phi(\tilde{y}(x))-\tilde{y}(x))^{T}
\end{array}\right],
$$

where $\tilde{y}^{T}(x) \triangleq\left[\begin{array}{ll}y^{T}(x) & \left(y^{+}(x)\right)^{T}\end{array}\right]$. In this case the relation between $x$ and $x^{+}$described by (7a) is taken into account.

Lemma 4. For any vector $\zeta \in \mathbb{R}^{n_{\zeta}}$ and matrix $R \in$ $\mathbb{R}^{n_{\zeta} \times\left(2 n_{y}+n\right)}$ the relation

$$
s_{4}(R, \zeta, x) \triangleq \zeta^{T} R Q \tilde{\chi}(x)=0
$$

with

$$
Q=\left[\begin{array}{c:cc:cc:cc}
f_{5} & F_{3} & 0 & F_{4}-I & 0 & I & 0 \\
f_{5} & 0 & F_{3} & 0 & F_{4}-I & 0 & I \\
0 & F_{1} & -I & F_{2} & 0 & 0 & 0
\end{array}\right]
$$

is verified along the trajectories of the closed-loop system (7).

Proof. Similarly to the proof of Lemma 3, note that from relations (7a) and (7b), we have $Q \tilde{\chi}(x)=0 \forall x \in \mathbb{R}^{n}$.

The next theorem gives sufficient conditions for the global exponential stability of the origin of system (7). For this, we consider a continuous piecewise quadratic (PWQ) Lyapunov candidate function generically described as follows:

$$
V(x)=\left[\begin{array}{c}
x \\
\phi(y)
\end{array}\right]^{T}\left[\begin{array}{cc}
P_{1} & P_{2} \\
P_{2}^{T} & P_{3}
\end{array}\right]\left[\begin{array}{c}
x \\
\phi(y)
\end{array}\right]=\left[\begin{array}{c}
x \\
\phi(y)
\end{array}\right]^{T} P\left[\begin{array}{c}
x \\
\phi(y)
\end{array}\right]
$$

with $P_{1} \in \mathbb{R}^{n \times n}, P_{2} \in \mathbb{R}^{n \times n_{y}}$ and $P_{3} \in \mathbb{R}^{n_{y} \times n_{y}}$.

Theorem 1. Consider a PWA system (7) with $f_{5} \preceq$ 0 and $V(x)$ as in (14). If there exist matrices $P=$ $P^{T} \in \mathbb{R}^{\left(n+n_{y}\right) \times\left(n+n_{y}\right)}, T_{1} \in \mathbb{D}^{n_{y}}, T_{2} \in \mathbb{D}^{2 n_{y}}, R_{1} \in$ $\mathbb{R}^{\left(1+n+2 n_{y}\right) \times n_{y}}, R_{2} \in \mathbb{R}^{\left(1+2 n+4 n_{y}\right) \times\left(2 n_{y}+n\right)}$, elementwise nonnegative matrices $M_{1} \in \mathbb{R}^{\left(1+2 n_{y}\right) \times\left(1+2 n_{y}\right)}$ and $M_{2} \in$ 
$\mathbb{R}^{\left(1+4 n_{y}\right) \times\left(1+4 n_{y}\right)}$ and positive scalars $\epsilon_{1}$ and $\eta \in(0,1)$ such that

$$
\begin{gathered}
\left(V(x)-\epsilon_{1} x^{T} x\right)+s_{3}\left(R_{1}, \chi(x), x\right)+s_{2}\left(T_{1}, y(x)\right) \\
-s_{1}\left(M_{1}, y(x)\right) \geq 0
\end{gathered}
$$

and

$$
\begin{gathered}
-\left(V\left(x^{+}\right)-\eta V(x)\right)+s_{4}\left(R_{2}, \tilde{\chi}(x), x\right)+s_{2}\left(T_{2}, \tilde{y}(x)\right) \\
-s_{1}\left(M_{2}, \tilde{y}(x)\right) \geq 0
\end{gathered}
$$

with $\chi(x), \tilde{y}(x)$ and $\tilde{\chi}(x)$ as previously defined, then the origin of system (7) is globally exponentially stable.

Proof. Since $f_{5}$ is assumed to be nonpositive, then $V(x)$ has a finite quadratic upper bound given by $\epsilon_{2}\|x\|^{2}$ [4]. Moreover, applying Lemmas 1, 2 and 3, inequality (15) implies that

$$
\epsilon_{1}\|x\|^{2} \leq V(x) \leq \epsilon_{2}\|x\|^{2} .
$$

On the other hand, from Lemmas 1, 2 and 4, inequality (16) implies that

$$
V\left(x^{+}\right) \leq \eta V(x) .
$$

Since $\eta \in(0,1)$ and $V(x)>0$, then $\Delta V(x) \triangleq$ $V\left(x^{+}\right)-V(x)<0$. Moreover, from (18), we conclude that $V(x(k)) \leq \eta^{k} V(x(0))$, which, from (17), implies that $\|x(k)\| \leq \sqrt{\epsilon_{2} / \epsilon_{1}} e^{k \ln (\sqrt{\eta})}\|x(0)\| \forall x(0) \in \mathbb{R}^{n}$, from where the global exponential stability of the origin follows.

The conditions (15) and (16) can be written in a matrix form. In this case, given $\eta \in(0,1)$, these conditions become linear matrix inequalities (LMIs) in variables $P, T_{1}, T_{2}, R_{1}$, $R_{2}, M_{1}, M_{2}$ and $\epsilon_{1}$ as stated in the following theorem.

Theorem 2. Given $\eta \in(0,1)$, if there exist matrices $P \in \mathbb{R}^{\left(n+n_{y}\right) \times\left(n+n_{y}\right)}, T_{1} \in \mathbb{D}^{n_{y}}, T_{2} \in \mathbb{D}^{2 n_{y}}, R_{1} \in$ $\mathbb{R}^{\left(1+n+2 n_{y}\right) \times n_{y}}, \quad R_{2} \in \mathbb{R}^{\left(1+2 n+4 n_{y}\right) \times\left(2 n_{y}+n\right)}, \quad M_{1} \in$ $\mathbb{R}^{\left(1+2 n_{y}\right) \times\left(1+2 n_{y}\right)}, M_{2} \in \mathbb{R}^{\left(1+4 n_{y}\right) \times\left(1+4 n_{y}\right)}$ and a positive scalar $\epsilon_{1}$ such that the LMIs

$$
\begin{aligned}
& {\left[\begin{array}{cccc}
0 & 0 & 0 & 0 \\
0 & P_{1}-\epsilon_{1} I & P_{2} & 0 \\
0 & P_{2}^{T} & P_{3} & 0 \\
0 & 0 & 0 & 0
\end{array}\right]-Z_{1}+\operatorname{He}\left\{R_{1} Q_{1}\right\} \geq 0} \\
& {\left[\begin{array}{c:cc:cc:cc}
0 & 0 & 0 & 0 & 0 & 0 & 0 \\
0 & \eta P_{1} & 0 & \eta P_{2} & 0 & 0 & 0 \\
0 & 0 & -P_{1} & 0 & -P_{2} & 0 & 0 \\
\hdashline 0 & \star & 0 & \eta P_{3} & 0 & 0 & 0 \\
0 & 0 & \star & 0 & -P_{3} & 0 & 0 \\
\hdashline 0 & 0 & 0 & 0 & 0 & 0 & 0 \\
0 & 0 & 0 & 0 & 0 & 0 & 0
\end{array}\right]-Z_{2}+\operatorname{He}\left\{R_{2} Q_{2}\right\} \geq 0}
\end{aligned}
$$

and the elementwise nonnegativity constraints

$$
M_{1} \succeq 0 \text { and } M_{2} \succeq 0
$$

with $Q_{1}=\left[\begin{array}{llll}f_{5} & F_{3} & F_{4}-I & I\end{array}\right], Q_{2}$ as defined in (13),

$$
Z_{1}=\left[\begin{array}{cccc}
M_{1_{11}} & 0 & M_{1_{12}} & M_{1_{13}} \\
0 & 0 & 0 & 0 \\
\star & 0 & M_{1_{22}} & M_{1_{23}}-T_{1} \\
\star & 0 & \star & M_{1_{33}}
\end{array}\right] \text { and }
$$

$$
Z_{2}=\left[\begin{array}{c:cc:c:c}
M_{211} & 0 & 0 & M_{2_{12}} & M_{2_{13}} \\
0 & 1 & 0 & 0 & 0 \\
0 & 0 & 0 & 0 & 0 \\
\hdashline \star & 0 & 0 & M_{22} & M_{223}-T_{2} \\
\hdashline \star & 0 & 0 & \star & M_{23}
\end{array}\right],
$$

are satisfied, then the origin of system (7) with $f_{5} \preceq 0$ is globally exponentially stable.

Proof. By pre and post multiplying (19) by $\chi^{T}(x)$ and $\chi(x)$, respectively, we obtain (15). Moreover, by pre and post multiplying (20) by $\tilde{\chi}^{T}(x)$ and $\tilde{\chi}(x)$, respectively, we obtain (16). Constraints (21) ensure the elementwise nonnegativity of matrices $M_{1}$ and $M_{2}$.

\section{Global Stabilization}

Although Theorem 2 is useful to assess the origin stability of a given CPWA system through a direct LMI feasibility test, the same cannot be done in the stabilization problem. This is due to the product between variables $R_{2}$ and $Q_{2}$. Note that $F_{1}$ and $F_{2}$, which are defined from $K_{1}$ and $K_{2}$, appear in matrix $Q_{2}$. To address this problem, we perform a congruence transformation and fix the structure of some matrices. The resulting stabilization condition is proposed in the Theorem below.

Theorem 3. Given $\eta \in(0,1)$, if there exist $\tilde{P}=\tilde{P}^{T} \in$ $\mathbb{R}^{\left(n+n_{y}\right) \times\left(n+n_{y}\right)}$, positive definite $\tilde{E}=\tilde{E}^{T} \in \mathbb{R}^{n \times n}, \tilde{M}_{1} \in$ $\mathbb{R}^{\left(1+2 n_{y}\right) \times\left(1+2 n_{y}\right)}, \tilde{T}_{1} \in \mathbb{D}^{n_{y}}, \quad \tilde{M}_{2} \in \mathbb{R}^{\left(1+4 n_{y}\right) \times\left(1+4 n_{y}\right)}$, $\tilde{T}_{2} \in \mathbb{D}^{2 n_{y}}$, non-singular symmetric matrices $W_{1} \in \mathbb{R}^{n \times n}$, $W_{2} \in \mathbb{D}^{n_{y}}, W_{3} \in \mathbb{D}^{n_{y}}, W_{4} \in \mathbb{D}^{n_{y}}$ and $W_{5} \in \mathbb{D}^{n_{y}}$, matrices $\tilde{K}_{1} \in \mathbb{R}^{n_{u} \times n}$ and $\tilde{K}_{2} \in \mathbb{R}^{n_{u} \times n_{y}}$ and scalars $\alpha, \beta$ and $\gamma$ such that the matrix inequalities

$$
\left[\begin{array}{cccc}
0 & 0 & 0 & 0 \\
0 & \tilde{P}_{1}-\tilde{E} & \tilde{P}_{2} & 0 \\
0 & \star & \tilde{P}_{3} & 0 \\
0 & 0 & 0 & 0
\end{array}\right]-\tilde{Z}_{1}+\operatorname{He}\left\{\tilde{R}_{1} \tilde{Q}_{1}\right\} \geq 0
$$

$$
\left[\begin{array}{c:cc:cc:cc}
0 & 0 & 0 & 0 & 0 & 0 & 0 \\
0 & \eta \tilde{P}_{1} & 0 & \eta \tilde{P}_{2} & 0 & 0 & 0 \\
0 & 0 & -\tilde{P}_{1} & 0 & -\tilde{P}_{2} & 0 & 0 \\
\hdashline 0 & \star & 0 & \eta P_{3} & 0 & 0 & 0 \\
0 & 0 & \star & 0 & -\tilde{P}_{3} & 0 & 0 \\
\hdashline 0 & 0 & 0 & 0 & 0 & 0 & 0
\end{array}\right]-\tilde{Z}_{2}+\operatorname{He}\left\{\tilde{R}_{2} \tilde{Q}_{2}\right\} \geq 0
$$

and the elementwise nonnegativity constraints

$$
\Pi_{1}^{-1}\left[\begin{array}{cccc}
\tilde{M}_{1_{11}} & 0 & \tilde{M}_{1_{12}} & \tilde{M}_{1_{13}} \\
0 & 0 & 0 & 0 \\
\star & 0 & \tilde{M}_{1_{22}} & \tilde{M}_{1_{23}} \\
\star & 0 & \star & \tilde{M}_{1_{33}}
\end{array}\right] \Pi_{1}^{-1} \succeq 0
$$

$$
\Pi_{2}^{-1}\left[\begin{array}{c:cc:c:c}
\tilde{M}_{2_{11}} & 0 & 0 & \tilde{M}_{2_{12}} & \tilde{M}_{2_{13}} \\
0 & 0 & 0 & 0 & 0 \\
0 & 0 & 0 & 0 & 0 \\
\hdashline \star & 0 & 0 & \tilde{M}_{22} & M_{223} \\
\hdashline \star & 0 & 0 & \star & \tilde{M}_{23}
\end{array}\right] \Pi_{2}^{-1} \succeq 0
$$


are satisfied with $\Pi_{1}=\Pi_{1}^{T}=\operatorname{diag}\left(1, W_{1}, W_{2}, W_{5}\right), \Pi_{2}=$ $\Pi_{2}^{T}=\operatorname{diag}\left(1, W_{1}, W_{1}, W_{2}, W_{2}, W_{3}, W_{4}\right)$,

$$
\begin{aligned}
& \tilde{Z}_{1}=\left[\begin{array}{cccc}
\tilde{M}_{1_{11}} & 0 & \tilde{M}_{1_{12}} & \tilde{M}_{1_{13}} \\
0 & 0 & 0 & 0 \\
\star & 0 & \tilde{M}_{1_{22}} & \tilde{M}_{1_{23}}-\tilde{T}_{1} \\
\star & 0 & \star & \tilde{M}_{1_{33}}
\end{array}\right], \tilde{R}_{1}=\left[\begin{array}{c}
0 \\
0 \\
\gamma I \\
I
\end{array}\right] \\
& \tilde{Q}_{1}=\left[\begin{array}{llll}
f_{5} & F_{3} W_{1} & \left(F_{4}-I\right) W_{2} & W_{5}
\end{array}\right] \text {, } \\
& \tilde{Z}_{2}=\left[\begin{array}{ccccc}
\tilde{M}_{2_{11}} & 0 & 0 & \tilde{M}_{2_{12}} & \tilde{M}_{2_{13}} \\
0 & 0 & 0 & 0 & 0 \\
0 & 0 & 0 & 0 & 0 \\
\star & 0 & 0 & \tilde{M}_{22} & \tilde{M}_{2_{23}}-\tilde{T}_{2} \\
\star & 0 & 0 & \star & \tilde{M}_{2_{33}}
\end{array}\right] \text {, } \\
& \tilde{R}_{2}=\left[\begin{array}{ccccccc}
0 & 0 & 0 & I & 0 & I & 0 \\
0 & 0 & 0 & 0 & \beta I & 0 & I \\
0 & I & \alpha I & 0 & 0 & 0 & 0
\end{array}\right]^{T} \text { and } \\
& \tilde{Q}_{2}^{T}=\left[\begin{array}{ccc}
f_{5}^{T} & f_{5}^{T} & 0 \\
\left(F_{3} W_{1}\right)^{T} & 0 & \left(F_{1 o} W_{1}+B \tilde{K}_{1}\right)^{T} \\
0 & \left(F_{3} W_{1}\right)^{T} & -W_{1} \\
\left(\left(F_{4}-I\right) W_{2}\right)^{T} & 0 & \left(F_{2 o} W_{2}+B \tilde{K}_{2}\right)^{T} \\
0 & \left(\left(F_{4}-I\right) W_{2}\right)^{T} & 0 \\
W_{3} & 0 & 0 \\
0 & W_{4} & 0
\end{array}\right],
\end{aligned}
$$

then the gains $K_{1}=\tilde{K}_{1} W_{1}^{-1}$ and $K_{2}=\tilde{K}_{2} W_{2}^{-1}$ ensure that the origin of the closed-loop system (7) is globally exponentially stable.

Proof. Starting with (20), consider the following particular structure for matrix $R_{2}$ :

$$
R_{2}=\left[\begin{array}{ccccccc}
0 & 0 & 0 & W_{2}^{-1} & 0 & W_{3}^{-1} & 0 \\
0 & 0 & 0 & 0 & \beta W_{2}^{-1} & 0 & W_{4}^{-1} \\
0 & W_{1}^{-1} & \alpha W_{1}^{-1} & 0 & 0 & 0 & 0
\end{array}\right]^{T}
$$

After pre and post multiplying (20) by the symmetric matrix $\Pi_{2}$ the term $\Pi_{2} R_{2} Q_{2} \Pi_{2}$ becomes $\tilde{R}_{2} \tilde{Q}_{2}$ considering the change of variables $\tilde{K}_{1} \triangleq K_{1} W_{1}$ and $\tilde{K}_{2} \triangleq K_{2} W_{2}$. The first term of (23) is obtained considering $\tilde{P}_{1} \triangleq W_{1} P_{1} W_{1}$, $\tilde{P}_{2} \triangleq W_{1} P_{2} W_{2}$ and $\tilde{P}_{3} \triangleq W_{2} P_{3} W_{2}$. Finally, the term $\tilde{Z}_{2}$ in (23) is obtained from the following change of variables.

$$
\begin{aligned}
\tilde{M}_{2_{11}} \triangleq M_{2_{11}}, \quad \tilde{T}_{2} \triangleq\left[\begin{array}{cc}
W_{2} & 0 \\
0 & W_{2}
\end{array}\right] T_{2}\left[\begin{array}{cc}
W_{3} & 0 \\
0 & W_{4}
\end{array}\right], \\
\tilde{M}_{2_{12}} \triangleq M_{2_{12}}\left[\begin{array}{cc}
W_{2} & 0 \\
0 & W_{2}
\end{array}\right], \quad \tilde{M}_{2_{13}} \triangleq M_{2_{13}}\left[\begin{array}{cc}
W_{3} & 0 \\
0 & W_{4}
\end{array}\right], \\
\tilde{M}_{2_{22}} \triangleq\left[\begin{array}{cc}
W_{2} & 0 \\
0 & W_{2}
\end{array}\right] M_{2_{22}}\left[\begin{array}{cc}
W_{2} & 0 \\
0 & W_{2}
\end{array}\right], \\
\tilde{M}_{2_{23}} \triangleq\left[\begin{array}{cc}
W_{2} & 0 \\
0 & W_{2}
\end{array}\right] M_{2_{23}}\left[\begin{array}{cc}
W_{3} & 0 \\
0 & W_{4}
\end{array}\right] \text { and } \\
\tilde{M}_{2_{33}} \triangleq\left[\begin{array}{cc}
W_{3} & 0 \\
0 & W_{4}
\end{array}\right] M_{2_{33}}\left[\begin{array}{cc}
W_{3} & 0 \\
0 & W_{4}
\end{array}\right] .
\end{aligned}
$$

Consider now (19), but replace the positive scalar $\epsilon_{1}$ by a positive definite matrix $E$. This procedure ensures that $V(x)$ is greater than a positive lower bound given by $\lambda_{\min }(E)\|x\|^{2}$, where $\lambda_{\min }(E)$ is the minimal eigenvalue of $E$, and allows for a change of variables. Moreover, consider the following structure for matrix $R_{1}=\left[\begin{array}{lllll}0 & 0 & \gamma W_{2}^{-1} & W_{5}^{-1}\end{array}\right]^{T}$. Then, after pre and post multiplying (19) by the symmetric matrix $\Pi_{1}$ the term $\Pi_{1} R_{1} Q_{1} \Pi_{1}$ becomes $\tilde{R}_{1} \tilde{Q}_{1}$ and the following change of variables is considered to obtain the remaining terms of (22):

$$
\begin{aligned}
& \tilde{E} \triangleq W_{1} E W_{1}, \quad \tilde{M}_{1_{11}} \triangleq M_{1_{11}}, \quad \tilde{M}_{1_{12}} \triangleq M_{1_{12}} W_{2} \\
& \tilde{M}_{1_{13}} \triangleq M_{1_{13}} W_{3}, \quad \tilde{M}_{1_{22}} \triangleq W_{2} M_{1_{22}} W_{2}, \quad \tilde{M}_{1_{23}} \triangleq W_{2} M_{1_{23}} W_{3} \\
& \tilde{M}_{1_{33}} \triangleq W_{3} M_{1_{33}} W_{3} \text { and } \tilde{T}_{1} \triangleq W_{2} T_{1} W_{3} .
\end{aligned}
$$

Finally, note that the constraints (24) and (25) ensure that the elementwise constraints in (21) are satisfied, i.e., $M_{1}$ and $M_{2}$ in Theorem 1 are nonnegative elementwise.

Note that conditions in Theorem 3 are nonconvex. There appear products between scalars $\alpha, \beta$ and $\gamma$ and some unknown matrices. Also, the elementwise constraints (24) and (25) include products of unknown matrices. In the next section, we propose an algorithm to solve the stabilization problem based on Theorem 3 using convex optimization.

\section{Proposed Algorithm}

Since constraints (22) to (25) are nonconvex, it is important to propose an algorithm to solve the feasibility problem defined by such constraints.

First, there is the product between variable matrices $\tilde{R}_{1} \tilde{Q}_{1}$ and $\tilde{R}_{2} \tilde{Q}_{2}$. Since matrices $\tilde{R}_{1}$ and $\tilde{R}_{2}$ have only a few scalar variables, then the gridding method proposed in [12] can be used, i.e., define a grid of values for $\alpha, \beta$ and $\gamma$ and, for each point in the grid, (22) and (23) are LMIs. The grid is characterized by a minimal value $\left(\alpha_{\text {min }}, \beta_{\text {min }}, \gamma_{\text {min }}\right)$, a step value $\left(\alpha_{s}, \beta_{s}, \gamma_{s}\right)$ and a maximum value $\left(\alpha_{\max }, \beta_{\max }\right.$, $\left.\gamma_{\max }\right)$ for each variable.

In addition to (22) and (23), we must also satisfy the elementwise constraints (24) and (25). Noting that $W_{2}, W_{3}$, $W_{4}$ and $W_{5}$ are diagonal matrices, we impose these matrices to be positive or negative definite and then add constraints on the corresponding elements of matrices $\tilde{M}_{1}$ and $\tilde{M}_{2}$. We have, therefore, 16 possible cases as described by Table I. For instance, consider case 3 (i.e. $W_{5}>0, W_{4}>0, W_{3}<0$, $\left.W_{2}<0\right)$. Thus, for this case, from (27) we must impose the following elementwise constraints $\tilde{M}_{1_{11}} \succeq 0, \tilde{M}_{1_{12}} \preceq 0$, $\tilde{M}_{1_{13}} \preceq 0, \tilde{M}_{1_{22}} \succeq 0, \tilde{M}_{1_{23}} \succeq 0$, and $\tilde{M}_{1_{33}} \succeq \overline{0}$ to ensure that matrix $M_{1}$ is elementwise nonnegative. The same procedure must be applied to $\tilde{M}_{2}$ following (26) to ensure that matrix $M_{2}$ is elementwise nonnegative.

Hence, the idea is to check the feasibility of (22) and (23) in a grid on $\alpha, \beta$ and $\gamma$, considering the elementwise contraints associated to each one of the cases in Table I.

Once a feasible solution is found, the stabilizing gains are given by $K_{1}=\tilde{K}_{1} W_{1}^{-1}$ and $K_{2}=\tilde{K}_{2} W_{2}^{-1}$, as stated in Theorem 3. Additional performance constraints, such as minimization of $\eta$ (i.e. maximization of the convergence rate), can be considered to choose the best pair of stabilizing gains among the feasible cases in Table I. 


\begin{tabular}{c|c|c|c|c|c|c|c|c|c}
\hline Case & $W_{5}$ & $W_{4}$ & $W_{3}$ & $W_{2}$ & Case & $W_{5}$ & $W_{4}$ & $W_{3}$ & $W_{2}$ \\
\hline 0 & $>$ & $>$ & $>$ & $>$ & 8 & $<$ & $>$ & $>$ & $>$ \\
\hline 1 & $>$ & $>$ & $>$ & $<$ & 9 & $<$ & $>$ & $>$ & $<$ \\
\hline 2 & $>$ & $>$ & $<$ & $>$ & 10 & $<$ & $>$ & $<$ & $>$ \\
\hline 3 & $>$ & $>$ & $<$ & $<$ & 11 & $<$ & $>$ & $<$ & $<$ \\
\hline 4 & $>$ & $<$ & $>$ & $>$ & 12 & $<$ & $<$ & $>$ & $>$ \\
\hline 5 & $>$ & $<$ & $>$ & $<$ & 13 & $<$ & $<$ & $>$ & $<$ \\
\hline 6 & $>$ & $<$ & $<$ & $>$ & 14 & $<$ & $<$ & $<$ & $>$ \\
\hline 7 & $>$ & $<$ & $<$ & $<$ & 15 & $<$ & $<$ & $<$ & $<$ \\
\hline
\end{tabular}

TABLE I

TABLE OF CASES TESTED FOR MATRICES $W_{2}$ TO $W_{5}$

\section{NUMERICAL EXAMPLE}

Consider a discrete-time approximation of the system presented in Section 8.1 of [10], obtained with $T=0.5$ and implicitly represented as in (2) with

$$
\begin{aligned}
& F_{1 o}=\left[\begin{array}{cc}
1 & T \\
4 T & 1-0.25 T
\end{array}\right], F_{2 o}=\left[\begin{array}{cccc}
0 & 0 & 0 & 0 \\
T & -T & -T & T
\end{array}\right], B=\left[\begin{array}{c}
0 \\
T
\end{array}\right] \\
& F_{3}=\left[\begin{array}{cc}
0 & 0.4024 \\
0 & 0.2638 \\
0 & -0.4024 \\
0 & -0.2638
\end{array}\right], F_{4}=0, \text { and } f_{5}=\left[\begin{array}{l}
-0.4024 \\
-1.3190 \\
-0.4024 \\
-1.3190
\end{array}\right] .
\end{aligned}
$$

This system locally approximates the nonlinear function given in [10] by the piecewise function described in Figure 3 .

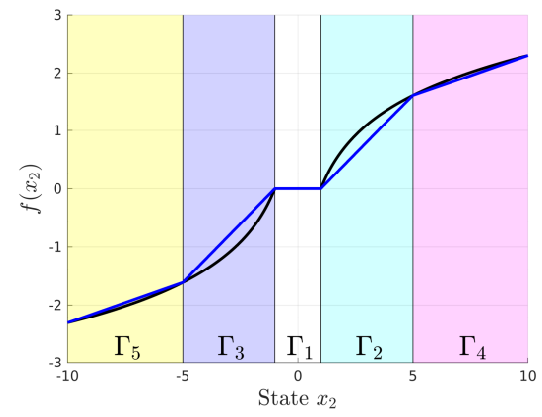

Fig. 3. Nonlinear function (black) and its PWA approximation (blue).

It should be noticed that the origin of the open-loop system (2) is not globally exponentially stable. Thus, applying the method proposed in Section VI with parameters $\eta=$ $0.99, \alpha_{\min }=-1.5, \alpha_{s}=0.5, \alpha_{\max }=1.5, \beta_{\min }=$ $-1.5, \beta_{s}=0.5, \beta_{\max }=1.5, \gamma_{\min }=-1.5, \gamma_{s}=$ 0.5 and $\gamma_{\max }=1.5$ results in the following global stabilizing gains $K_{1}=\left[\begin{array}{ll}-5.2350 & -3.9165\end{array}\right]$ and $K_{2}=$ $\left[\begin{array}{llll}-0.1614 & 0.1243 & 0.1625 & -0.1242\end{array}\right]$ for $\alpha=1.5, \beta=$ $1.0, \gamma=1.5$ and test case 1 . Some closed-loop trajectories are shown in Figure 4.

\section{CONCLUSION}

This work addressed the stabilization of discrete-time continuous piecewise affine systems written in a recently proposed implicit representation. This representation was reviewed and sufficient conditions for global stabilization were derived. An algorithm, based on the solution of LMI feasibility problems, was proposed to obtain a

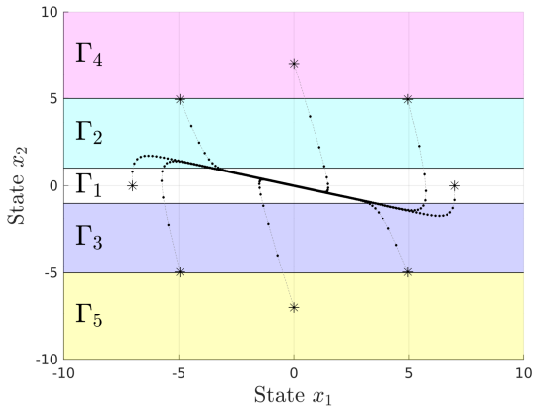

Fig. 4. Example 1: examples of closed-loop trajectories.

stabilizing nonlinear feedback law. Differently from previous approaches in the literature, the enumeration of transitions is not needed and the presence of the affine term is taken into account without further difficulties. Future work shall include the case where the partition is modified by the control law and applications regarding neural networks with ReLU (i.e. ramp) activation functions.

\section{REFERENCES}

[1] A. Bemporad, F. D. Torrisi, and M. Morari. Optimization-based verification and stability characterization of piecewise affine and hybrid systems. In Hybrid Systems: Computation and Control, pages 45-58. Springer Berlin Heidelberg, 2000.

[2] G. Feng. Stability analysis of piecewise discrete-time linear systems. IEEE Transactions on Automatic Control, 47(7):1108-1112, 2002.

[3] G. Ferrari-Trecate, F. A. Cuzzola, D. Mignone, and M. Morari. Analysis of discrete-time piecewise affine and hybrid systems. Automatica, 38(12):2139 - 2146, 2002.

[4] L. B. Groff, G. Valmorbida, and J. M. Gomes da Silva Jr. Stability analysis of piecewise affine discrete-time systems. In Proceedings of the 58th IEEE Conference on Decision and Control (CDC), pages 8172-8177, 2019.

[5] A. Hassibi and S. Boyd. Quadratic stabilization and control of piecewise-linear systems. In Proceedings of the 1998 American Control Conference, volume 6, pages 3659-3664, 1998.

[6] W. P. M. H. Heemels, B. De Schutter, and A. Bemporad. Equivalence of hybrid dynamical models. Automatica, 37(7):1085 - 1091, 2001.

[7] M. Johansson. Piecewise Linear Control Systems. Springer-Verlag Berlin Heidelberg, 2003.

[8] H. K. Khalil. Nonlinear Systems; 3rd ed. Prentice-Hall, 2002.

[9] D. Mignone, G. Ferrari-Trecate, and M. Morari. Stability and stabilization of piecewise affine and hybrid systems: an LMI approach. In Proceedings of the 39th IEEE Conference on Decision and Control $(C D C)$, pages 504-509, 2000.

[10] L. G. Moreira, J. M. Gomes da Silva Jr., S. Tarbouriech, and A. Seuret. Observer-based event-triggered control for systems with slope-restricted nonlinearity. International Journal of Robust and Nonlinear Control, 30(17):7409-7428, 2020.

[11] J. A. Primbs and M. Giannelli. Kuhn-tucker-based stability conditions for systems with saturation. IEEE Transactions on Automatic Control, 46(10):1643-1647, 2001.

[12] L. Rodrigues and S. Boyd. Piecewise-affine state feedback for piecewise-affine slab systems using convex optimization. Systems and Control Letters, 54(9):835-853, 2005.

[13] E. Sontag. Nonlinear regulation: The piecewise linear approach. IEEE Transactions on Automatic Control, 26(2):346-358, 1981.

[14] K. Zou and S. Nagarajaiah. Study of a piecewise linear dynamic system with negative and positive stiffness. Communications in Nonlinear Science and Numerical Simulation, 22(1):1084-1101, 2015. 